\title{
Darier Disease following Blaschko Lines- Case Report
}

\author{
G Pehlivanov ${ }^{1}$, N Tsekova Traykovich ${ }^{1}$, I Bakardzhiev ${ }^{2}$, R Pavlova $^{1}$, M Balabanova ${ }^{1}$, L Miteva ${ }^{1}$, \\ ${ }^{1}$ Department of Dermatology and Venerology, Medical University-Sofia, Bulgaria \\ ${ }^{2}$ Medical College, Medical University- Varna, Bulgaria
}

Received: July 14, 2016; Accepted: July 25, 2016; Published: August 01, 2016

*Corresponding author: Assoc. Prof. Ilko Bakardzhiev, Medical College, Medical University of Varna, Tsar Osvo boditel 84, Bulgaria, Tel: +359 888768 413; E-Mail: varna2008@gmail.com

\begin{abstract}
Darier Disease (DD), or keratosis follicularis, is an uncommon, slowly progressive, autosomal-dominant skin disorder. DD is characterized clinically by multiple keratotic papules and histological finding shows loss of adhesion between epidermal cells and abnormal keratinization.

We describe an unusual case of late-onset unilateral segmental DD which follows the lines of Blaschko. A 63-year-old woman without comorbidities was presented to dermatology department with a 10-years history of a mildly pruritic unilateral " zosteriform" rash on her trunk. The patient was treated in the past for Herpes zoster several times without improvement. No nail or oral mucosa involvement were established.

Results of a biopsy of lower abdominal and gluteal lesions showed foci of suprabasilar acantholysis and dyskeratosis including both corps ronds and grains. The patient was treated with topical retinoids.

The type 1 segmental manifestation reflects a de novo postzygotic somatic mutation in the heterozygous state and as a rule can be found in all autosomal dominantly inherited genodermatoses.
\end{abstract}

Keywords: Darier Diseases; Unilateral “Zosteriform” Rash; type 1

\section{Case Report}

A 63-year-old woman without comorbidities was presented in dermatology department with a 10-years history of a mildly pruritic unilateral "zosteriform" rash on her trunk. The patient was previously treated for Herpes zoster infection with Acyclovir $5 \times 800 \mathrm{mg}$ without improvement in the appearance and pruritus of the rash. She noted that no one else in her family had such a condition.

Physical examination revealed scattered, 1- to 3-mm, erythematous to light-brown papules with scale in swirling distribution along the Blaschko lines beginning at the patient's abdominal midline, continuing over her left flank, and extending partially onto her mid back and gluteal area. Multi linear separate areas delineated by a band of unaffected skin were apparent. (Fig. $1,2,3,4)$. No nail or oral mucosa involvement were observed.

The biopsy specimen of lower abdominal and gluteal lesions showed foci of suprabasilar acantholysis and dyskeratosis

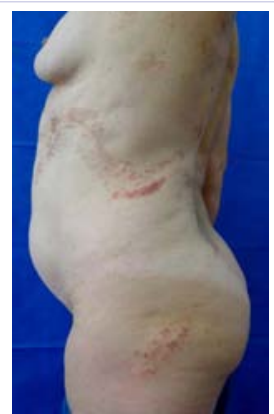

Figure 1: Scattered, erythematous to light-brown papules with scale in swirling distribution along the Blaschko lines.

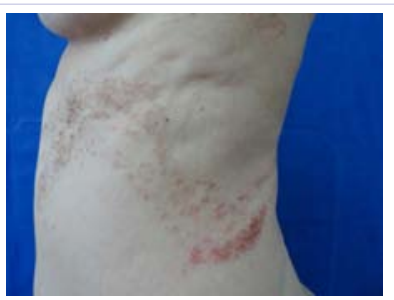

Figure 2: Scattered, erythematous to light-brown papules with scale over left half of the body.

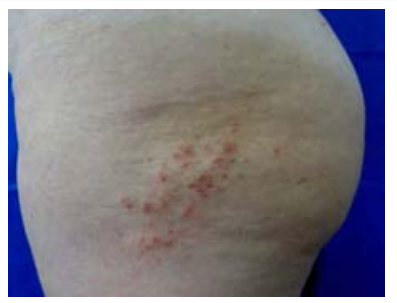

Figure 3: Scattered, erythematous to light-brown papules with scale over left thigh.

including both "corps ronds" and "grains". (Fig. 5,6,). The patient was treated with topical retinoids (Isotertionin cr. $0.1 \%$ ) with strict follow-up.

\section{Discussion}

Darier Disease (DD) was first reported in 1889 independently by French dermatologist Jean Darier and Proff. James White from 


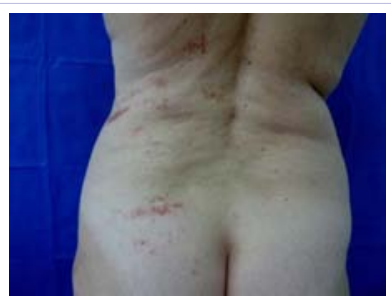

Figure 4: Scattered, erythematous to light-brown papules with scale over mid back and left gluteal area.

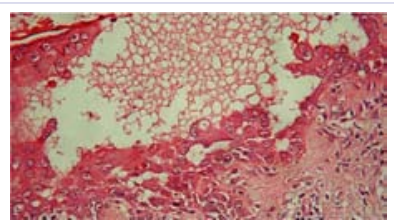

Figure 5: Foci of suprabasilar acantholysis and dyskeratosis including both "corps ronds" and "grains".

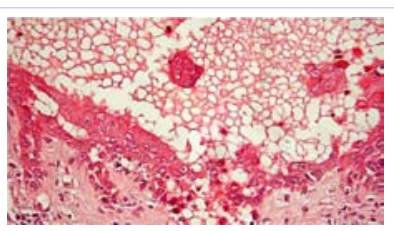

Figure 6: "Corps ronds" and "grains".

Harvard University. It was White who recognized genetic nature of keratosis follicularis by noticing the daughter of his initially described patient developed similar skin lesions. Clinically, the disease is characterized by greasy, yellow to brown crusted papules which develop mainly in seborrheic areas of head, neck and trunk. In $10 \%$ of cases of DD there is localized distribution [1]. Localized pattern was first reported by Kreibich in 1906 [2]. Since then, several variants of the localized disease have been observed: unilateral, linear, segmental or zosteriform. In this localized subtypes skin lesions are confined to a limited area, but the involved skin has the same mutations in ATP2A2 gene that occur in the generalized form of the disease. Two types of segmental variants of DD have been observed. The more common type I is characterized by unilateral distribution along Blashko's lines. The skin surrounding the affected zone is normal. This distribution is a result from genetic mosaicism due to postzygotic mutations and not as an example of epidermal nevus as previously thought. It is now well known that autosomal dominant disease may sometimes become manifest in a mosaic form, which involve skin in circumscribed, patchy or, like in this case, linear arrangement. If there is associated gonadal mosaicism, a patient with segmental manifestations may have offspring with generalized disease. In the type II of segmental variant there is generalized DD with linear streak of increased severity [3]. Type II mosaicism occurs in patients with a heterozygous germline mutations who also have a somatic loss of heterozygosity of wild type allele in a segmental area leading to homo- or hemirozygosity and therefore increased severity in a linear array. Accordingly, the linear pattern presented in our case, would represent type I segmental
DD. In localized forms is often absent of other mucocutaneous characteristics which are presented in widespread disease (nail, palm or mucosa membrane changes). The onset of the disease is usually in the third or fourth decade of life. This is older age compared to than observed in generalized form (early adolescence) [4]. The differential diagnoses of segmented DD include herpes zoster, linear nevoid disorders, lichen striatus, and lichen planus. The diagnose is confirmed by biopsy with histopathologycal examination. The histological characters of suprabasal acantholysis with typical "corps ronds" and "grains" are the clues for diagnosis. Corps ronds' are acantholytic enlarged keratinocytes in the malpighian layer with darkly staining and partially fragmented nuclei surrounded by a clear cytoplasm and encircled by a bright ring of collapsed keratin bundles. 'Grains' are small, oval cells in the stratum corneum characterized by an intensely eosinophilic cytoplasm composed of collapsed keratin bundles containing shrunken parakeratotic nuclear remnants. Daily skin care includes the use of antimicrobial cleansers, emollients, use of sun protection creames and moisturizers with urea or lactic acid. Topical corticosteroids as monotherapy are often less effective than topical retinoids. For systemic treatment is commonly used systemic retinoids and oral antibiotics. COX2 inhibitors are possible therapeutic strategy based on one in vitro study which showed that COX-2 inhibition may restore down regulation of ATP2A2/SERCA2 expression in keratinocytes caused by UVB irradiation $[5,6]$.

\section{Conclusion}

We present a case of unilateral DD which follows the Blaschko lines treated successfully with isotretionin cr. $0.1 \%$ and discuss the differential diagnoses. The unilateral presentation is due to a genetic mosaicism that results during embryologic development. Although it is very rare entity, we should keep this diagnosis in our minds and do not confuse with other differential diagnosis.

\section{References}

1. Lin FL, Hu SL, Liang CW. Seasonal recurrence of linear Darier's disease. Dermatol Sinica. 2008;26:236-241.

2. Barfield RL, Barrett KR, Moon CM, David-Bajar K. Pruritic linear papules on a 75 year old woman: a case of localized Darier-White disease. Cutis. 2002;70(4):225-228.

3. Happle R, Itin PH, Brun AM. Type 2 segmental manifestation of Darier's disease. Eur J Dermatol. 1999;9(6):449-451.

4. O’Malley MP, Haake A, Goldsmith L, Berg D. Localized Darier's disease. Implications for genetic studies. Arch Dermatol. 1997;133(9):11341138.

5. Gilaberte M, Puig L, Vidal D, Alomar A. Acantholytic dyskeratotic naevi following Blaschko's lines: a mosaic form of Darier's disease. J Eur Acad Dermatol Venereol. 2003;17(2):196-199.

6. Kamijo M, Nishiyama C, Takagi A, Nakano N, Hara M, Ikeda S, et al. Cyclooxygenase-2 inhibition restores ultraviolet B-induced downregulation of ATP2A2/SERCA2 in keratinocytes: possible therapeutic approach of cyclooxygenase-2 inhibition for treatment of Darier disease. Br J Dermatol. 2012;166(5):1017-1022. doi: 10.1111/j.1365-2133.2011.10789.x. 